\title{
THE RADIAL HEAT POLYNOMIALS AND RELATED FUNCTIONS
}

\author{
BY \\ L. R. BRAGG(1)
}

1. Introduction. In a lengthy paper by Rosenbloom and Widder [9] and a more recent paper by Widder [12], a detailed study has been made of necessary and sufficient conditions for the validity of expansions of solutions of the heat equation

$$
\frac{\partial u(x, t)}{\partial t}=\frac{\partial^{2} u(x, t)}{\partial x^{2}}
$$

in terms of two basic sets of special solutions: (a) the set $\left\{v_{j}(x, t)\right\}_{j=0}^{\infty}$ of heat polynomials and (b) the associated set $\left\{w_{j}(x, t)\right\}_{j=0}^{\infty}$. The elements of the first set are defined symbolically and analytically by

$$
v_{j}(x, t)=e^{t D_{x}^{2}} \cdot x^{j}=(-t)^{j / 2} H_{j}\left(x /(-4 t)^{1 / 2}\right), \quad j=0,1,2, \cdots,
$$

where $H_{j}(x)$ is the usual Hermite polynomial. The element $w_{j}(x, t)$ of the second set is obtained by forming the Appell transform of the $v_{j}(x, t)$. For $t>0$, the two sets $\left\{v_{j}(x,-t)\right\}$ and $\left\{w_{j}(x, t)\right\}$ are biorthogonal on $(-\infty, \infty)$. The basic theorems relating to the expansion of solutions of (1.1) show that

(I) expansions in terms of the heat polynomials are valid in a time strip in which the solution satisfies a Huygen's principle while

(II) expansions in terms of the associated functions are valid in an upper halfplane $(t>\sigma \geqq 0)$ in which the solution has certain entireness properties. At the same time, these theorems give rules for determining the coefficients in these expansions both by real and complex evaluations. The $L^{2}$ theory of such expansions is then examined. Of basic importance in getting at these results are (i) the decomposition of the fundamental source solution of (1.1), $S(x, t)=(4 \pi t)^{-1 / 2} e^{-x^{2} / 4 t}$, in terms of these basic heat functions and (ii) the equivalence of the important Huygen's principle to an absolute integrability condition on solutions of (1.1).

In this paper, we will be concerned with the generalized heat equation

$$
\frac{\partial u(r, t)}{\partial t}=\Delta_{\mu} \cdot u(r, t)
$$

where $\mu=2(\alpha+1), \alpha>-\frac{1}{2}$, and $\Delta_{\mu} \equiv \partial^{2} / \partial r^{2}+(\mu-1) / r \partial / \partial r$. The operator $\Delta_{\mu}$ is

Presented to the Society, January 27, 1965; received by the editors October 1, 1964.

(1) This research was supported by the National Aeronautics and Space Administration, Grant Number NsG-544. 
the Laplacian in radial coordinates when $\mu=n$, a positive integer. Of particular interest will be the obtaining of results analogous to (I) and (II) involving expansions of solutions of (1.3) in terms of the two basic sets of functions related to (1.3), the radial heat polynomials $\left\{R_{j}^{\mu}(r, t)\right\}_{j=0}^{\infty}$ and their Appell transforms $\left\{\tilde{R}_{j}^{\mu}(r, t)\right\}_{j=0}^{\infty}$. These sets are defined in terms of the generalized Laguerre polynomials $L_{j}^{(x)}(x)$. Moreover, for $t>0$, the set $\left\{R_{j}^{\mu}(r,-t)\right\}$ is biorthogonal to the set $\left\{\widetilde{R}_{j}^{\mu}(r, t)\right\}$ on $[0, \infty)$ relative to a suitable weight factor. Unlike the treatment in [9], the solution of (1.3) subject to $u(r, 0)=\phi(r)$ has an integral representation with a more complicated kernel (involving Bessel functions). Nevertheless, we are able to give a decomposition of this kernel in terms of the sets $\left\{R_{j}^{\mu}\right\}$ and $\left\{\widetilde{R}_{j}^{\mu}\right\}$ and examine the indicated type of theorems relating to representations of solutions of (1.3). The restriction $\alpha>-\frac{1}{2}$ is taken for simplicity because of the structure of the asymptotic bounds on the Laguerre polynomials. The important case $\alpha=-\frac{1}{2}$ can be readily reduced to the theory in [9] by means of the connection between the functions $L_{j}^{(-1 / 2)}\left(x^{2}\right)$ and $H_{2 j}(x)$.

We make no attempt to be as detailed or complete in our treatment as were Rosenbloom and Widder. For example, we omit the complex evaluation of coefficients in expansions as well as the $L^{2}$ theory of such expansions. Rather, emphasis is placed on the differences arising from the use of Laguerre polynomials. These differences assert themselves in the bounds on the basic sets of functions, the kernel of the integral representation of solutions of (1.3), and the decomposition of this kernel. To avoid a repetition with [9], we state a theorem that equates the Huygen's principle to an absolute integrability condition. This equivalence is made evident by reducing the proof of the existence of a Poisson-Stieltjes integral representation for certain solutions of (1.3) to the same types of arguments used in [9]. This is handled by indicating the validity of the Tychonoff theorem for solutions of (1.3) and by establishing suitable growth bounds on such solutions.

It will, at times, be convenient to consider $(1.3)$ when $2(\alpha+1)=n$, a positive integer. For this situation, the decomposition $\Delta_{n}=D_{1}^{2}+\cdots+D_{n}^{2}, D_{i} \equiv \partial / \partial x_{i}$, for rectangular coordinates will be useful in motivating relations between various functions. Some of these will be meaningful even if $n$ fails to be an integer. We occasionally use symbolic operators, such as $\exp \left(t \Delta_{\mu}\right)$, for expressing solutions of (1.3) and their properties in a convenient form. We interpret this operator by

$$
\exp \left(t \Delta_{\mu}\right) \cdot \phi(r)=\sum_{j=0}^{\infty} \frac{t^{j}}{j !} \Delta_{\mu}^{j} \cdot \phi(r)
$$

whenever the series in (1.4) is meaningful. Otherwise, we interpret this operator by means of an integral to be given in $\S 3$.

2. The radial heat functions. Let $S_{\mu}(r, t)$ denote the fundamental source solution of (1.3), that is

$$
S_{\mu}(r, t)=(4 \pi t)^{-\mu / 2} e^{-r^{2} / 4 t}, \quad \mu=2(\alpha+1)
$$


Let $u(r, t)$ be a solution of (1.3) in some domain $\mathscr{D}(u)$. Then if $(r / t,-1 / t) \in \mathscr{D}(u)$, we define the Appell transform [1] $\tilde{u}$ of $u$ by

$$
\tilde{u}(r, t)=S_{\mu}(r, t) u(r / t,-1 / t) .
$$

In terms of this, we can specify the basic set of functions. Let $k$ be a non-negative integer. We define the radial heat polynomial $R_{k}^{\mu}(r, t)$ as the analytic solution of (1.3) subject to the condition $u(r, 0)=r^{2 k}$ and its associated function $\widetilde{R}_{k}^{\mu}(r, t)$ as the Appell transform of $R_{k}^{\mu}(r, t)$. One can easily give explicit forms for these. By (1.4),

$$
\begin{aligned}
R_{k}^{\mu}(r, t) & =\exp \left(t \Delta_{\mu}\right) r^{2 k} \\
& =\Gamma\left(\frac{\mu}{2}+k\right) \sum_{j=0}^{k} \frac{1}{\Gamma\left(\frac{\mid \mu}{2}+j\right)}\left(\begin{array}{l}
k \\
j
\end{array}\right) r^{2 j}(4 t)^{k-j}
\end{aligned}
$$

and from this one readily obtains $\widetilde{R}_{k}^{\mu}(r, t)$. Although we will not use this information, it will be noted that $\exp \left(t \Delta_{\mu}\right) \cdot r^{2 k}$, when interpreted by (1.4), is meaningful for any complex $\mu$ and $R_{k}^{\mu}(r, t)$ is a continuous function of $\mu$. Observe from (2.3) that for $r \geqq 0$ and $t>0$,

$$
R_{k}^{\mu}(r, t) \geqq \frac{\Gamma\left(\frac{\mu}{2}+k\right)}{\Gamma\left(\frac{\mu}{2}\right)}(4 t)^{k}>0, \quad k=0,1,2, \cdots .
$$

For the purpose of getting at further properties of these basic sets of heat functions, such as biorthogonality, let us now examine their connection with the generalized Laguerre polynomials by means of a generating function. One such generating function is immediately available for the radial heat polynomials. For if we require $u_{a}(r, 0)=e^{a r^{2}}$ to be satisfied by the analytic solution of (1.3), then it can be checked directly in (1.3) that

$$
u_{a}(r, t)=(1-4 a t)^{-\mu / 2} \exp \left(a r^{2} /(1-4 a t)\right), \quad 1-4 a t>0 .
$$

A comparison of this with the generating function for the Laguerre polynomials $\left[4\right.$, p. 189] shows that by making the choices $T=4 a t$ and $X=-r^{2} / 4 t$ we have

$$
u_{a}(r, t)=\sum_{j=0}^{\infty} T^{j} L_{j}^{(\alpha)}(X)=\sum_{j=0}^{\infty}(4 t)^{j} L_{j}^{(\alpha)}\left(-r^{2} / 4 t\right) a^{j} .
$$

In addition, we have formally

$$
\exp \left(t \Delta_{\mu}\right) \cdot e^{a r^{2}}=\sum_{j=0}^{\infty} \frac{a^{j}}{j !} \exp \left(t \Delta_{\mu}\right) \cdot r^{2 J}=\sum_{j=0}^{\infty} \frac{1}{j !} R_{j}^{\mu}(r, t) a^{j}
$$


A comparison of (2.6) with (2.7) then shows that

$$
R_{j}^{\mu}(r, t)=j !(4 t)^{j} L_{j}^{(\alpha)}\left(-r^{2} / 4 t\right), \quad j=0,1,2, \cdots
$$

and this is the desired connection with the Laguerre polynomials. From the form of the source solution, the following development results:

$$
S_{\mu}(r, t)=(4 \pi)^{-\mu / 2} \sum_{j=0}^{\infty} \frac{(4)^{-j}}{j !} R_{j}^{\mu}(i r, 1-t), \quad t>0 .
$$

By an application of the definitions (2.2) and (2.3), we find the functions associated with the radial heat polynomials to be

$$
\widetilde{R}_{j}^{\mu}(r, t)=t^{-2 j} S_{\mu}(r, t) R_{j}^{\mu}(r,-t), \quad j=0,1,2, \cdots
$$

when $(r / t,-1 / t)$ is in the domain $\mathscr{D}\left(R_{j}^{\mu}\right)$. A generating function, $\tilde{u}_{a}(r, t)$, for these is given by

$$
\tilde{u}_{a}(r, t)=S_{\mu}(r, t+4 a)=\sum_{j=0}^{\infty} \frac{a^{j}}{j !} \tilde{R}_{j}^{\mu}(r, t), \quad t+4 a \neq 0 .
$$

From the fact that $S_{\mu}(r, t)$ satisfies the semi-group property

$$
S_{\mu}(r, t+4 a)=\exp \left(4 a \Delta_{\mu}\right) \cdot S_{\mu}(r, t)
$$

(see Theorem (6.4b) and (2.11)) it follows that

$$
\tilde{R}_{j}^{\mu}(r, t)=\left.\frac{\partial^{j}}{\partial a^{j}} S_{\mu}(r, t+4 a)\right|_{a=0}=\left(4 \Delta_{\mu}\right)^{j} S_{\mu}(r, t) .
$$

The author uses this relation to obtain a Rodrigues' formula for the Laguerre polynomials that involves the Laplacian operator [2].

Next, from the orthogonality relations for the generalized Laguerre polynomials [8, p. 205], it follows by (2.8) and (2.10) that for $t>0$,

$$
\int_{0}^{\infty} W_{\mu}(r) R_{j}^{\mu}(r,-t) \tilde{R}_{k}^{\mu}(r, t) d r= \begin{cases}0 & \text { if } j \neq k, \\ j ! 2^{4 j} \Gamma\left(\frac{\mu}{2}+j\right) & \text { if } j=k\end{cases}
$$

where

$$
W_{\mu}(r)=2\{\pi\}^{\mu / 2} r^{\mu-1} .
$$

This is the biorthogonality relation for the radial heat functions which permits a determination of the coefficients in expansions of solutions of (1.3) in terms of these basic functions.

Finally, when $\mu$ is a positive integer $n$, we relate these radial heat polynomials to the usual heat polynomials. We do so by a symbolic approach. Let $r^{2}=x_{1}^{2}+\cdots+x_{n}^{2}$ and write $\Delta_{n}=D_{1}^{2}+\cdots+D_{n}^{2}, D_{j}=\partial / \partial x_{j}$. With the factorization $\exp \left(t \Delta_{n}\right)=\prod_{j=1}^{n} \exp \left(t D_{j}^{2}\right)$, it follows by (1.2) and (2.3) that 


$$
\begin{aligned}
R_{k}^{n}(r, t) & =\exp \left(t \Delta_{n}\right) r^{2 k}=\exp \left(t \Delta_{n}\right)\left(x_{1}^{2}+\cdots+x_{n}^{2}\right)^{k} \\
& =\sum_{k_{1}+\ldots+k_{n}=k ; k_{i} \geqq 0}\left(\begin{array}{c}
k \\
k_{1}, k_{2}, \cdots, k_{n}
\end{array}\right) \prod_{j=1}^{n} e^{t D^{j}} x_{j}^{2 k_{j}} \\
& =\sum_{k_{1}+\ldots+k_{k}=k ; k: \geqq 0}\left(\begin{array}{c}
k \\
k_{1}, k_{2}, \cdots, k_{n}
\end{array}\right) \prod_{j=1}^{n} v_{2 k_{j}}\left(x_{j}, t\right),
\end{aligned}
$$

with

$$
\left(\begin{array}{c}
k \\
k_{1}, k_{2}, \cdots, k_{n}
\end{array}\right)
$$

the usual multinomial coefficient. The choice $t=-1 / 4$ in this gives a result of Feldheim [5] when $n=2$.

3. The integral representation for the solution. Let us now interpret the operation $\exp \left(t \Delta_{\mu}\right) \cdot \phi(r)$ for more general $\phi(r)$ than that allowable by (1.4). This can be accomplished by transforming the Poisson formula in $\mu=n$ dimensional space in rectangular coordinates to the radially symmetric form. The final result permits an interpretation even if $\mu$ fails to be an integer. We simply outline the derivation here.

Let $\bar{r}$ denote the $n$-vector $\left(r_{1}, \cdots, r_{n}\right)$ and let $\bar{r} \circ \bar{\xi}$ denote the scalar product $\sum_{i=1}^{n} r_{i} \cdot \xi_{i}$. Let $r=(\bar{r} \circ \bar{r})^{1 / 2}, \xi=(\bar{\xi} \circ \bar{\xi})^{1 / 2}$, and $d \bar{\xi}=d \xi_{1} \cdots d \xi_{n}$. Now the Poisson formula in rectangular coordinates is just

$$
u(\bar{r}, t)=(4 \pi t)^{-n / 2} \int_{E_{n}} \phi(\bar{\xi}) \exp \left[-\frac{1}{4 t}(\bar{r}-\bar{\xi}) \circ(\bar{r}-\bar{\xi})\right] d \bar{\xi}
$$

where $E_{n}$ denotes all of $n$-space. Let $\theta_{1}$ denote the angle between the vectors $\bar{r}$ and $\xi$ so that $(\bar{r}-\bar{\xi}) \circ(\bar{r}-\bar{\xi})^{1 / 2}=r^{2}+\xi^{2}-2 r \xi \cos \theta_{1}$. Taking $n \geqq 2$, we introduce the spherical coordinates $\left(\xi, \theta_{1}, \cdots, \theta_{n-1}\right)$ in (3.1) with $0 \leqq \xi<\infty$, $0 \leqq \theta_{i} \leqq \pi, i=1, \cdots, n-2$, and $0 \leqq \theta_{n-1} \leqq 2 \pi$. The Jacobian of this transformation is $J=\xi^{n-1} \sin ^{n-2} \theta_{1} \sin ^{n-3} \theta_{2} \cdots \sin \theta_{n-2}$ and we must evaluate the integral

$$
\int_{0}^{2 \pi} \int_{0}^{\pi} \cdots \int_{0}^{\pi}\left\{\exp \left[\frac{r \xi}{2 t} \cos \theta_{1}\right] \sin ^{n-2} \theta_{1}\right\} \sin ^{n-3} \theta_{2} \cdots \sin \theta_{n-2} d \theta_{1} \cdots d \theta_{n-1}
$$

By successive reductions of this through use of the Beta function and the definition of the modified Bessel function (3.1) reduces to

$$
u(r, t)=\int_{0}^{\infty} K_{n}(r, \xi ; t) \phi(\xi) d \xi
$$

with the kernel

$$
K_{n}(r, \xi ; t)=\frac{1}{2 t} r^{1-(n / 2)} \xi^{n / 2} e^{-\left(r^{2}+\xi^{2}\right) / 4 t} I_{n / 2-1}\left(\frac{r \xi}{2 t}\right) .
$$

Of course, suitable continuity and growth restrictions are needed on $\phi(\xi)$ in 
(3.2) to ensure that the integral converges. We can now replace $n$ by $\mu$ in (3.2) and (3.3). For future reference, we note that for $t>0$,

$$
\lim _{r \rightarrow 0} K_{\mu}(r, \xi ; t)=W_{\mu}(\xi) S_{\mu}(\xi, t)
$$

(b)

$$
\lim _{\xi \rightarrow 0} K_{\mu}(r, \xi ; t)= \begin{cases}0 & \text { if } \mu>1, \\ (4 \pi)^{1 / 2} S_{1}(r, t) & \text { if } \mu=1 .\end{cases}
$$

From the first of these, we can thus attach a meaning to $K_{\mu}(0, \xi ; t)$.

In order to develop representations of solutions of (1.3) in terms of the sets $\left\{R_{j}^{\mu}\right\}$ and $\left\{\tilde{R}_{j}^{\mu}\right\}$, it will be useful to examine the structure of $K_{\mu}(r, \xi ; t)$ in terms of these functions. This, however, will necessitate deriving some elementary properties of the kernel relating to these basic functions along with the development of asymptotic bounds for these basic functions. We defer the latter question to the next section.

THEOREM 3.1. Let $r \geqq 0$ and $t>0$. Then

$$
R_{j}^{\mu}(r,-t)=\int_{0}^{\infty} K_{\mu}(i r, \xi ; t)(i \xi)^{2 j} d \xi
$$

(b)

$$
R_{j}^{\mu}(r, t)=\int_{0}^{\infty} K_{\mu}(r, \xi ; t) \xi^{2 j} d \xi
$$

Proof. Now from (3.3) we find

$$
K_{\mu}(i r, \xi ; t)=\frac{1}{2 t} r^{1-\mu / 2} \xi^{\mu / 2} e^{\left(r^{2}-\xi^{2}\right) / 4 t} J_{\mu / 2-1}\left(\frac{r \xi}{2 t}\right)
$$

and thus the right-member of (3.5a) becomes

$$
\frac{(-1)^{j}}{2 t} r^{1-\mu / 2} e^{r^{2} / 4 t} \int_{0}^{\infty} \xi^{\mu / 2+2 j} e^{-\xi^{2} / 4 t} J_{\mu / 2-1}\left(\frac{r \xi}{2 t}\right) d \xi .
$$

The change of variables $s=\xi^{2} / 4 t$ along with an application of the result of equation (5), p. 135 of [3] (which also yields (3.5a) with $r$ replaced by $-i r$ ) shows that this last integral reduces to $(-4 t)^{j} j ! L_{j}^{(\mu / 2-1)}\left(r^{2} / 4 t\right)$. By (2.8), this gives (3.5a). From the validity of the result for - ir in (3.5a), we get

$$
\begin{aligned}
R_{j}^{\mu}(r, t) & =R_{j}^{\mu}(i[-i r], t)=(-1)^{j} R_{j}^{\mu}(-i r, t) \\
& =(-1)^{j} \int_{0}^{\infty} K_{\mu}\left(-i^{2} r, \xi ; t\right)(i \xi)^{2 j} d \xi \\
& =\int_{0}^{\infty} K_{\mu}(r, \xi ; t) \xi^{2 j} d \xi .
\end{aligned}
$$

This is just the representation (3.5b). 
Next, we examine the important time translation property.

THEOREM 3.2. Let $-s<t<s$ and let $r \geqq 0, \eta \geqq 0$. Then

$$
\begin{aligned}
& \text { (a) } K_{\mu}(r, \eta ; s+t)=\int_{0}^{\infty} K_{\mu}(r, \xi ; t) K_{\mu}(\xi, \eta ; s) d \xi, \quad t>0, \\
& \text { (b) } K_{\mu}(r, \eta ; s+t)=\int_{0}^{\infty} K_{\mu}(i r, \xi ;-t) K_{\mu}(i \xi, \eta ; s) d \xi, \quad t<0 .
\end{aligned}
$$

Proof. Both results follow through an application of the formula (25), p. 50 of [4] for Bessel functions. The terms in the integrand of (3.6b) involve the functions $J_{\mu / 2-1}$ with non-negative arguments so that the indicated formula applies directly. The proof of (3.6a) can be reduced to the use of this same formula with complex arguments through use of the relation $I_{\alpha}(r \xi / 2 t)=e^{-i \alpha \pi / 2} J_{\alpha}\left(r \xi e^{i \pi / 2} / 2 t\right)$, $\alpha=\mu / 2-1$.

Finally, we note for later reference the interchange property

$$
K_{\mu}(r, \xi ; t)=(\xi / r)^{\mu-1} K_{\mu}(\xi, r ; t) .
$$

4. Growth bounds and the kernel decomposition. Let us now examine growth bounds for the radial heat functions. In decomposing the kernel (3.3) in terms of such functions, we will need to know in what sense such a decomposition converges. The bounds we obtain depend upon the connection between these basic functions and the Laguerre polynomials. We need some of the elementary results obtained in [9].

LEMMA 1. If $0 \leqq r<\infty, 0 \leqq t<\infty$, and $0<\delta<\infty, k=0,1,2, \cdots$, then it follows that

$$
\left|R_{k}^{\mu}(r, t)\right| \leqq\left(1+\frac{t}{\delta}\right)^{\mu / 2}\left\{\frac{4 k(t+\delta)}{e}\right\}^{k} e^{r^{2} / 4 \delta}
$$

Now, it is known that if $\alpha \geqq 0$ and $x \geqq 0$, then $\left|L_{j}^{(\alpha)}(x)\right| \leqq(\alpha+1)_{j}(i !)^{-1} e^{x / 2}$ (see [4, p. 207]) with $(\alpha+1)_{j}=\prod_{k=1}^{j}(\alpha+k)$. Then by $(2.8)$

$$
\left|R_{k}^{\mu}(r,-t)\right| \leqq \frac{(4 t)^{k} \Gamma\left(\frac{\mu}{2}+k\right) e^{r^{2} / 8 t}}{\Gamma(\mu / 2)}
$$

By an application of Stirling's formula, there exists a constant $A>0$ such that for all $k$

$$
\Gamma(\mu / 2+k) \leqq A\left(\frac{\mu / 2+k}{e}\right)^{\mu / 2+k}[2 \pi(k+\mu / 2)]^{1 / 2}
$$

and we get the result: 
LEMMA II. For $r \geqq 0, t>0$, there exists a constant $A$ such that

(a) $\left|R_{k}^{\mu}(r,-t)\right| \leqq A\left\{\frac{4 t(\mu / 2+k)}{e}\right\}^{k}\left\{\frac{\mu}{2}+k\right\}^{(\mu+1) / 2} e^{r^{2} / 8 t}$,

(b) $\left|\tilde{R}_{k}^{\mu}(r, t)\right| \leqq A(4 \pi t)^{-\mu / 2}\left\{\frac{4(\mu / 2+k)}{t e}\right\}^{k}\left\{\frac{\mu}{2}+k\right\}^{(\mu+1) / 2} e^{-r^{2} / 4 t}$.

Further, let us note that since $I_{\alpha}(r) \leqq\left(\frac{1}{2} r\right)^{\alpha} e^{r} / \Gamma(\alpha+1)$ when $\alpha>-\frac{1}{2}$ and $r>0$ (p. 14 of [4], formula (4)), we have

LEMMA III. If $t>0, r>0$, and $\xi>0$, then

$$
K_{\mu}(r, \xi ; t) \leqq \frac{2 \xi^{\mu-1}}{(4 t)^{\mu / 2} \Gamma(\mu / 2)} e^{-(r-\xi)^{2} / 4} .
$$

We now have sufficient information to get at the desired decomposition of $K_{\mu}(r, \eta ; t+s)$. A further growth bound on the radial polynomials will be given 1ater.

LEMMA IV. Let $s>0, \xi \geqq 0$, and $\eta \geqq 0$. Then we have

$$
K_{\mu}(\xi, \eta ; s)=W_{\mu}(\eta) \sum_{l=0}^{\infty} \frac{\tilde{R}_{l}^{\mu}(\eta, s) \xi^{2 l}}{2^{4 l} l ! \Gamma\left(\frac{\mu}{2}+l\right)} .
$$

The replacement of $\xi$ by $i \xi$ in this also leads to a correct result.

Proof. This results from collecting the coefficients of like powers of $\xi$ in the series expansion for $K_{\mu}(\xi, \eta ; s)$. Thus

$$
\begin{aligned}
K_{\mu}(\xi, \eta ; s)= & S_{\mu}(\eta, s) W_{\mu}(\eta) e^{-\xi^{2} / 4 s} \sum_{l=0}^{\infty} \frac{\left(\frac{\xi \eta}{4 s}\right)^{2 l}}{l ! \Gamma(l+\mu / 2)} \\
= & S_{\mu}(\eta, s) W_{\mu}(\eta) \sum_{l, m=0}^{\infty} \frac{(-1)^{m} \eta^{2 l} \xi^{2(l+m)}}{l ! m ! \Gamma\left(\frac{\mu}{2}+l\right)(4 s)^{m+2 l}} \\
= & W_{\mu}(\eta) \sum_{j=0}^{\infty} \frac{\xi^{2 j}}{j ! 2^{4 j} \Gamma\left(j+\frac{\mu}{2}\right)} \\
& \cdot\left\{(-1)^{j} S_{\mu}(\eta, s) \Gamma\left(j+\frac{\mu}{2}\right) \sum_{l=0}^{j} \frac{(-1)^{l}\left(\begin{array}{l}
j \\
l
\end{array}\right) \eta^{2 l} 4^{j-l}}{s^{j+l} \Gamma\left(\frac{\mu}{2}+l\right)}\right) \\
= & W_{\mu}(\eta) \sum_{j=0}^{\infty} \frac{\tilde{R}_{j}^{\mu}(\eta, s) \xi^{2 j}}{2^{4 j} j ! \Gamma\left(\frac{\mu}{2}+j\right)} .
\end{aligned}
$$


The reduction to $\widetilde{R}_{j}^{\mu}(\eta, s)$ of the bracketed term in the next to the last series follows directly from (2.3) and the remark following it. The result with $\xi$ replaced by $i \xi$ holds since all functions involved here are entire for $s>0$.

We now come to the main decomposition theorem for $K_{\mu}$.

THEOREM 4.1. Let $s>0$ and $|t|<s$. If $r, \eta \geqq 0$, we have

$$
K_{\mu}(r, \eta ; t+s)=W_{\mu}(\eta) \cdot \sum_{l=0}^{\infty} \frac{R_{l}^{\mu}(r, t) \tilde{R}_{l}^{\mu}(\eta, s)}{2^{4 l} l ! \Gamma\left(\frac{\mu}{2}+l\right)} .
$$

Proof. We consider two cases according as (a) $t>0$ or (b) $t<0$.

Case (a). From the relation (3.6a) and (4.4) we have

$$
\begin{aligned}
K_{\mu}(r, \eta ; t+s) & =\int_{0}^{\infty} K_{\mu}(r, \xi ; t)\left\{W_{\mu}(\eta) \sum_{l=0}^{\infty} \frac{\tilde{R}_{l}^{\mu}(\eta, s) \xi^{2 l}}{2^{4 l} l ! \Gamma\left(\frac{\mu}{2}+l\right)}\right\} d \xi \\
& =W_{\mu}(\eta) \sum_{l=0}^{\infty} \frac{\tilde{R}_{l}^{\mu}(\eta, s)}{2^{4 l} l ! \Gamma\left(\frac{\mu}{2}+l\right)} \int_{0}^{\infty} K_{\mu}(r, \xi ; t) \xi^{2 l} d \xi
\end{aligned}
$$

and the result follows by $(3.5 b)$ provided that, say,

$$
\left|W_{\mu}(\eta)\right| \sum_{l=0}^{\infty} \frac{\left|\tilde{R}_{l}^{\mu}(\eta, s)\right|}{2^{4 l} l ! \Gamma\left(\frac{\mu}{2}+l\right)} \int_{0}^{\infty}\left|K_{\mu}(r, \xi ; t)\right| \cdot \xi^{2 l} d \xi<\infty .
$$

Introducing the bounds given in (4.1) and (4.2), this series is dominated by the sum

$$
W_{\mu}(\eta) \cdot A(4 \pi s)^{-\mu / 2} e^{-\eta^{2} / 8 s+r^{2} / 4 \delta}(1+t / \delta)^{\mu / 2} \cdot \sigma
$$

where

$$
\sigma=\sum_{l=0}^{\infty} \frac{\left\{\frac{4(\mu / 2+l)}{s e}\right\}^{l}\left\{\frac{\mu}{2}+l\right\}^{(\mu+1) / 2}\left\{\frac{4 l(t+\delta)}{e}\right\}^{l}}{2^{4 l} l ! \Gamma\left(\frac{\mu}{2}+l\right)}
$$

$0<\delta<s-t$. The limit of the ratio of the $(l+1)$ st to the $l$ th term in this sum $\sigma$ is just $(t+\delta) / s$ so that the theorem follows for $s>t \geqq 0$.

Case (b). From (3.6b) and the stated modification of (4.4), we find (for $s>|t|$ and $t<0$ ) 


$$
\begin{aligned}
K_{\mu}(r, \eta ; s+t) & =\int_{0}^{\infty} K_{\mu}(i r, \xi ;-t)\left\{W_{\mu}(\eta) \sum_{l=0}^{\infty} \frac{\tilde{R}_{l}^{\mu}(\eta, s)(i \xi)^{2 l}}{2^{4 l} l ! \Gamma\left(\frac{\mu}{2}+l\right)}\right\} d \xi \\
& =W_{\mu}(\eta) \sum_{l=0}^{\infty} \frac{\tilde{R}_{l}^{\mu}(\eta, s)}{2^{4 l} l ! \Gamma\left(\frac{\mu}{2}+l\right)} \int_{0}^{\infty} K_{\mu}(i r, \xi ;-t)(i \xi)^{2 l} d \xi
\end{aligned}
$$

and the result follows by $(3.5 \mathrm{a})$ (since $-t>0)$ provided that

But

$$
W_{\mu}(\eta) \sum_{l=0}^{\infty} \frac{\left|\tilde{R}_{l}^{\mu}(\eta, s)\right|}{2^{4} l l ! \Gamma(l+\mu / 2)} \int_{0}^{\infty}\left|K_{\mu}(i r, \xi ;-t)\right| \xi^{2 l} d \xi<\infty . !
$$

$$
\begin{aligned}
\int_{0}^{\infty}\left|K_{\mu}(i r, \xi ; t)\right| \xi^{2 l} d \xi & =\int_{0}^{\infty}\left|\frac{1}{2 t} r^{1-\mu / 2} \xi^{\mu / 2} J_{\mu / 2-1}\left(\frac{r \xi}{2 t}\right)\right| \xi^{2 l} d \xi \\
& \leqq e^{r^{2} / 2 t} \int_{0}^{\infty} K_{\mu}(r, \xi ; t) \xi^{2 l} d \xi=e^{r^{2} / 2 t} R_{\mu}^{l}(r, t)
\end{aligned}
$$

Now, by the argument as used in Case (a), we get convergence of the dominating series.

We can now make an application of (3.7) along with (4.5) to give further decompositions. Using the same hypotheses as those in Theorem 4.1, we can interchange the arguments $r$ and $\eta$ under the sign of summation in the right member of (4.5). The factor $W_{\mu}(\eta)$ remains unaltered in this.

Finally, let us note from (2.8) and (2.10) that the relation (4.5) reduces to

$$
K_{\mu}(r, \eta ; t+s)=W_{\mu}(\eta) S_{\mu}(\eta, s) \sum_{l=0}^{\infty} \frac{l !}{\Gamma\left(\frac{\mu}{2}+l\right)} L_{l}^{(\alpha)}\left(\frac{-r^{2}}{4 t}\right) L_{l}^{(\alpha)}\left(\frac{\eta^{2}}{4 s}\right)\left(\frac{-t}{s}\right)^{l}
$$

for $|t|<s$ and $\mu=2(\alpha+1)$. Compare this with the relation (20), p. 189 of [4].

5. The coefficient behavior in convergent expansions. In this section we state results relating to the behavior of the coefficients involved in convergent expansions in terms of the sets $\left\{R_{j}^{\mu}(r, t)\right\}$ and $\left\{\tilde{R}_{j}^{\mu}(r, t)\right\}$. Such knowledge permits a characterization of the regions of validity of certain types of expansions in terms of constants related to an entire function (the order and the type). The methods for proving many of these results are similar to those in [9] but depend upon different bounds. In most cases, these proofs are omitted except where a difference arises because of the different underlying set of basic functions.

Lemma 5.1. Suppose that the series $\sum_{j=0}^{\infty} a_{j} R_{j}^{\mu}(r, t)$ converges at $\left(r_{0}, t_{0}\right)$ for $r_{0}>0, t_{0}>0$. Then 


$$
a_{j}=O\left\{\left[\frac{e}{4 t_{0}\{\mu / 2-1+j\}}\right]^{j+(\mu-1) / 2}\right\} \text { as } j \rightarrow \infty .
$$

Proof. By the assumed convergence of the series at $\left(r_{0}, t_{0}\right)$, it results that there exists a positive constant $M$ such that, for all $j,\left|a_{j} R_{j}^{\mu}\left(r_{0}, t_{0}\right)\right| \leqq M$. Then for all sufficiently large $j$,

$$
\begin{aligned}
\left|a_{j}\right| & \leqq M\left\{R_{j}^{\mu}\left(r_{0}, t_{0}\right)\right\}^{-1} \leqq \frac{M \Gamma(\mu / 2)}{\Gamma\left(\frac{\mu}{2}+j\right)\left(4 t_{0}\right)^{j}} \text { by (2.4) } \\
& \leqq M^{*}\left[\frac{4 t_{0}}{e}\left\{\frac{\mu}{2}-1+j\right\}\right]^{-((\mu-1) / 2+j)}, \quad M^{*}=M^{*}\left(\mu, t_{0}\right) .
\end{aligned}
$$

We have just made use of Stirling's formula in the last step. This final bound is just a restatement of (5.1). The lower bounds by (2.4) have the same form regardless of the parity of $k$ in $R_{k}^{\mu}(r, t)$. The forms of these lower bounds change in the case of the usual heat polynomials $v_{k}(x, t)$ according to the evenness or oddness of $k$. Thus, we get a simplification here.

REMARK. Since $\mu \geqq 2$, it follows that we could obtain the larger bound of $M^{*}\left[e /\left(4 t_{0} j\right)\right]^{j}$ for $\left|a_{j}\right|$. This larger and simpler expressed bound will prove to be useful later when converse results are given.

Our next result on the behavior of the coefficients $a_{j}$ depends upon the Fejér's asymptotic formula for the generalized Laguerre polynomials [10]. Let $x \in[\varepsilon, \omega]$, $\varepsilon>0$. Then as $j \rightarrow \infty$,

$L_{j}^{(\alpha)}(x)=\pi^{-1 / 2} e^{x / 2} x^{-(2 \alpha+1) / 4} j^{(2 \alpha-1) / 4} \cos \left[2(j x)^{1 / 2}-\left(\frac{2 \alpha+1}{4}\right) \pi\right]+O\left(j^{(2 \alpha-3) / 4}\right)$,

the error bound holding uniformly for all $x>0$. Through use of the connection (2.8) we have

LEMMA. 5.2. Let $t>0$ and restrict $r^{2} / 4 t$ to some finite interval $[\varepsilon, \omega], \varepsilon>0$. Then as $j \rightarrow \infty$,

$$
\begin{aligned}
& R_{j}^{\mu}(r,-t) \\
& =(-1)^{j} 4^{1 / 4} e^{(2 \alpha-3) / 4} r^{-\alpha-1 / 2} e^{r^{2} / 8 t}\left\{\frac{4 t j}{e}\right\}^{j+\alpha / 2+1 / 4} \\
& \cdot \cos \left[\left(j r^{2} / t\right)^{1 / 2}-\left(\frac{2 \alpha+1}{4}\right) \pi\right]+O\left(j^{(2 \alpha-3) / 4}\right),
\end{aligned}
$$

the error bound again holding uniformly for $r^{2} / 4 t>0$.

With this, we can immediately obtain 
LEMMA 5.3. Let $t_{0}>0$ and $0<\varepsilon_{1} \leqq a \leqq r \leqq b$ and suppose that $\sum_{j=0}^{\infty} a_{j} R_{j}^{\mu}\left(r,-t_{0}\right)$ converges for $r \in[a, b]$. Then $a_{j}=O\left[\left(e / 4\left|t_{0}\right| j\right)^{j+(\mu-1) / 4}\right]$.

THEOREM 5.1. Let $\left\{a_{j}\right\}_{j=0}^{\infty}$ be a sequence of complex numbers such that $\limsup \operatorname{sum}_{j \rightarrow \infty}\left\{\left|a_{j}\right|^{1 / j} 4 j / e\right\}=1 / \sigma<\infty$. Then the series $\sum_{j=0}^{\infty} a_{j} R_{j}^{\mu}(r, t)$ converges absolutely in the time strip $|t|<\sigma$ and not everywhere in any larger strip. This strip size is independent of $\mu$.

Proof. This follows by a dominated convergence proof by using the bounds on $\left|a_{j}\right|$ in the hypothesis and the bound (4.1) if $t \geqq 0$ and the bound (4.2a) if $t$ is negative.

Definition 5.1. An entire function is of growth $(\rho, \tau)$ iff

$$
\limsup _{j \rightarrow \infty}\left(\frac{j}{e \rho}\right)\left|a_{j}\right|^{\rho / j} \leqq \tau
$$

Definition 5.2. A function $u(r, t)$ satisfies condition $H$ in a domain $\mathscr{D}$ if $u(r, t)$ is continuous and satisfies (1.3) in $\mathscr{D}$.

THEOREM 5.2. Let $\sum_{j=0}^{\infty} a_{j} R_{j}^{\mu}(r, t)$ converge to some function $u(r, t)$ for $|t|<\sigma$. Then $u(r, t) \in H$ in this strip and $u(r, 0)$ is an even function which is entire with growth $(1,1 / 4 \sigma)$ in $r^{2}$.

Proof. The uniform convergence of the series to a solution function $u(r, t)$ follows directly by the usual application of Harnack's theorem for temperature functions [11] only modified to the $R_{j}^{\mu}(r, t)$. At $t=0$, this series converges to

$$
u(r, 0)=\sum_{j=0}^{\infty} a_{j} r^{2 j}
$$

an even function of $r$. Let $t_{0}$ be selected such that $\left|t_{0}\right|<\sigma$. Then $\left|a_{j}\right| \leqq M\left\{e / 4\left|t_{0}\right| j\right\}^{j+(\mu-1) / 4}$ for $j$ suitably large and

$$
\limsup _{j \rightarrow \infty} \frac{j}{e}\left|a_{j}\right|^{1 / j} \leqq \limsup _{j \rightarrow \infty} \frac{j}{e} M^{1 / j}\left\{\frac{e}{4\left|t_{0}\right| j}\right\}^{1+(\mu-1) / j}=\frac{1}{4\left|t_{0}\right|}
$$

Now let $\left|t_{0}\right| \rightarrow \sigma$ to get the theorem.

We also have the analogous theorems holding for expansions in terms of the $\tilde{R}_{j}^{\mu}(r, t)$.

THEOREM 5.3. If $\lim \sup _{j \rightarrow \infty} 4 j / e\left|b_{j}\right|^{1 / j}=\sigma<\infty$, then the series $\sum_{j=0}^{\infty} b_{j} \widetilde{R}_{j}^{\mu}(r, t)$ converges absolutely in the half-plane $t>\sigma$ and not everywhere in any including strip.

THEOREM 5.4. Let $\sum_{j=0}^{\infty} b_{j} \widetilde{R}_{j}^{\mu}(r, t)$ converge for $t>\sigma \geqq 0$. Then $u(r, t) \in H$ in this half-plane. 
6. Expansion theory preliminaries. In the previous sections, we have given most of the essential background for the formal work in developing expansions of solutions of (1.3) in terms of the sets $\left\{R_{j}^{\mu}\right\}$ and $\left\{\widetilde{R}_{j}^{\mu}\right\}$. Not all of these expansions are meaningful, however. As an example, when $\mu=3$, the function $1 / r, r \neq 0$, satisfies (1.3) for all $t$. This function has a formal expansion in terms of the set $\left\{R_{j}^{3}(r, t)\right\}$. If such an expansion converges at $t=0$, it converges to an even function, which would be the wrong function, and if the series diverges at $t=0$, the expansion is meaningless there.

We therefore need to be able to select appropriate classes of solutions of (1.3) each member $u(r, t)$ of which can be represented by a series of these basic heat functions which converges uniformly in some domain to $u(r, t)$. Of necessity, these will be analytic in $r^{2}$ for $r \geqq 0$ in the appropriate time range (in $n$-space, $n$ integer and $n \geqq 2$ if $r^{2}=x_{1}^{2}+\cdots+x_{n}^{2}$, then only even powers of $r$ are analytic in $\left(x_{1}, \cdots, x_{n}\right)$ in a neighborhood of the origin). In order to determine these classes, we give the following definitions.

Definition 6.1 (The Huygen's PRINCIPLe). A function $u(r, t)$ satisfies the Huygen's principle for $r \geqq 0$ and $a<t<b$, and we write $u \in H^{*}$, if $u \in H$ in this domain and $u$ satisfies the semi-group property

$$
u(r, t)=\int_{0}^{\infty} K_{\mu}\left(r, \xi ; t-t^{\prime}\right) u\left(\xi, t^{\prime}\right) d \xi \quad \text { for } a<t^{\prime}<t<b .
$$

Definition 6.2 (ABSOlute INTEgRABILITy CONDITION). A function $u(r, t)$ satisfies the condition of absolute integrability for $r \geqq 0$ and $a<t<b$, and we write $u \in A$, iff $u \in H$ and there exists a positive function $M\left(a^{\prime}, b^{\prime}\right)$ defined for $a<a^{\prime}<b^{\prime}<b$ such that

$$
\int_{0}^{\infty}|u(\xi, t)| W_{\mu}(\xi) S_{\mu}\left(\xi, b^{\prime}-t\right) d \xi<M\left(a^{\prime}, b^{\prime}\right), \quad a^{\prime}<t<b^{\prime} .
$$

The basic result relating these definitions that appears to be essential in the expansion theory is given by

THEOREM 6.1. Let $\mathscr{D}$ denote the domain $r \geqq 0$, $a<t<b$. Then $u \in H^{*}$ in $\mathscr{D}$ if and only if $u \in A$ in $\mathscr{D}$.

The analogous theorem is proved in detail in [9] and is based primarily on the existence of a Poisson-Stieltjes integral representation for certain absolutely integrable solutions of (1.1). Two important results used in this proof are (a) every solution of (1.1) that grows no more rapidly than $K e^{A x^{2}}, K$ and $A$ nonnegative constants, for $a<t<b$ satisfies the Huygen's principle over the part of this time range in which the corresponding integral converges absolutely (Lemma 6.2 of [9]) and (b) every absolutely integrable solution of (1.3) is bounded in growth by such an exponential function. The proof of (a) is based upon the Tychonoff 
theorem ([7, p. 183]) while (b) is based upon growth bounds related to the kernel involved in integral representations of solutions of (1.1). If we can establish the analogous results (a) and (b) for the problem involving (1.3) with corresponding kernel $K_{\mu}(r, \xi ; t)$, then we can use the reasoning of [9] to prove our Theorem 6.1. We lead up to these results through a sequence of elementary lemmas.

Lemma 6.1. Let $R>0$ and let $D_{R}$ denote the rectangle $0<r<R$ and $0<t \leqq c$. Let $B=\bar{D}_{R}-D_{R}$ be the boundary where $\bar{D}_{R}$ is the closed rectangle $0 \leqq r \leqq R, 0 \leqq t \leqq c$. Then if (i) $u(r, t) \in H$ in $D_{R}$ and (ii) $\liminf _{r \rightarrow r_{0} ; t \rightarrow t_{0}} u(r, t) \geqq 0$ for $\left(r_{0}, t_{0}\right) \in B$, it follows that $u(r, t) \geqq 0$ in $D_{R}$.

Proof. The argument needed here is similar to that in the proof of Theorem 5.2, p. 184 of [7]. The omission of the segment $r=0$ from the domain $D_{R}$ is required because of the singularity in the equation (1.3) at $r=0$. Also, if $\mu=3$, the function $1 / r$ fails to satisfy condition (i) at $r=0$ but does satisfy the conclusion of the lemma.

Lemma 6.2. Let $x \geqq 0$ and let $\mu>1$. Then we have

$$
x^{1 / 2} e^{-x} I_{\mu / 2-1}(x) \geqq b_{\mu} \int_{0}^{x} \eta^{(\mu-3) / 2} e^{-\eta} d \eta
$$

where $b_{\mu}=a_{\mu} /\left[2^{\mu / 2-1} \Gamma(1 / 2) \Gamma((\mu-1) / 2)\right]$ with $a_{\mu}=1$ if $\mu \geqq 3$ and $a_{\mu}=2^{(\mu-3) / 2}$ if $1<\mu<3$. The right member of (6.3) is a monotone increasing function of $x$ which has the finite positive limit $b_{\mu} \Gamma((\mu-1) / 2)$.

Proof. By exercise 16, p. 121 of [8], we have

$$
\begin{aligned}
I_{\mu / 2-1}(x) & =\frac{x^{\mu / 2-1}}{2^{\mu / 2-2} \Gamma\left(\frac{1}{2}\right) \Gamma\left(\frac{\mu-1}{2}\right)} \int_{0}^{\pi / 2} \sin ^{2(\mu / 2-1)} \theta \cosh (x \cos \theta) d \theta \\
& =\frac{x^{\mu / 2-1}}{2^{\mu / 2-2} \Gamma\left(\frac{1}{2}\right) \Gamma\left(\frac{\mu-1}{2}\right)} \int_{0}^{1}\left(1-s^{2}\right)^{(\mu-3) / 2} \cosh s x d s .
\end{aligned}
$$

The factor $(1+s)^{(\mu-3) / 2}$ of the integrand of this last integral has the lower bound $a_{\mu}$ on $[0,1]$. Then

$$
x^{1 / 2} e^{-x} I_{\mu / 2-1}(x) \geqq \frac{x^{(\mu-1) / 2} a_{\mu}}{2^{\mu / 2-1} \Gamma\left(\frac{1}{2}\right) \Gamma\left(\frac{\mu-1}{2}\right)} \int_{0}^{1}(1-s)^{(\mu-3) / 2} e^{-(1-s) x} d s
$$

where we have used the fact that $\cosh s x \geqq e^{s x} / 2$. The result follows from this last inequality by the change of variables $(1-s) x=\xi$.

From this, we have the direct consequence 
Lemma 6.3. Let $R \geqq 0$ and let $0<t \leqq c$. Then we have

$$
K_{\mu}(R, R ; t) \geqq(2 c)^{-1 / 2} b_{\mu} \int_{0}^{R^{2} / 2 c} \eta^{(\mu-3) / 2} e^{-\eta} d \eta
$$

Lemma 6.4 (Modified Tychonoff TheOREM). Let the following conditions be satisfied: (i) $u(r, t) \in H$ in $0<t \leqq c$ for $r>0$; (ii) $u(0+, t)=0,0<t \leqq c$; (iii) $\lim _{r \rightarrow r_{0} ; t \rightarrow 0_{+}} u(r, t)=0$ for all $r_{0}, 0 \leqq r_{0}<\infty$; (iv) $f(r)=\max _{0<t \leqq c}|u(r, t)|$; and $(\mathrm{v}) f(r)=O\left(e^{a r^{2}}\right)$ as $r \rightarrow \infty$ for some a. Then $u(r, t)=0$ throughout the strip $0<t \leqq c, r \geqq 0$.

Proof. Let $R>0$ and define the auxiliary function $U_{R}^{\mu}(r, t)$ :

$$
U_{R}^{\mu}(r, t)=\frac{(2 c)^{1 / 2} K_{\mu}(r, R ; t)}{b_{\mu} A(R)} f(R)
$$

with $A(R)=\int_{0}^{R^{2} / 2 c} \eta^{(\mu-3) / 2} e^{-\eta} d \eta$. Then by hypotheses, Lemma 6.3, and Lemma 6.1, it follows that the pair $U_{R}^{\mu}(r, t) \pm u(r, t) \geqq 0$ in $D_{R}$ or $-U_{R}^{\mu}(r, t) \leqq u(r, t) \leqq U_{R}^{\mu}(r, t)$ in $D_{R}$. Fix $r<R$ and let $R \rightarrow \infty$. By application of the growth bound (4.3), we have

$$
U_{R}^{\mu}(r, t) \leqq \frac{(8 c)^{1 / 2} R^{\mu-1}}{b_{\mu}(4 t)^{\mu / 2} \Gamma\left(\frac{\mu}{2}\right) A(R)} e^{-(r-R)^{2} / 4 t} f(R) .
$$

Since $A(R)$ tends to a finite nonzero limit as $R \rightarrow \infty$, it follows that $U_{R}^{\mu}(r, t) \rightarrow 0$ provided $4 a t<1$. If $c$ is in this time range, the proof is complete. If $c$ is not in this range, replace the condition (iii) at $t=0$ by the same condition at $t=b, b>0$ and $4 a b<1$. Then repeat the above argument a sufficient number of times.

We now make use of the following inequality, noted in [9], namely: if $A, x$, and $y$ are real numbers in $(-\infty, \infty), 0<t<\infty$, and $1-4 A t>0$, then

$$
A y^{2}-\frac{(y-x)^{2}}{4 t} \leqq \frac{A x^{2}}{1-4 A t} .
$$

LemMa 6.5. Let $t^{\prime}<t<t_{0}$. Then we have

$$
\begin{aligned}
K_{\mu}\left(r, \xi ; t-t^{\prime}\right) / K_{\mu}( & \left.r_{0}, \xi ; t_{0}-t^{\prime}\right) \\
& \leqq \Gamma\left(\frac{\mu}{2}\right)\left(\frac{t_{0}-t^{\prime}}{t-t^{\prime}}\right)^{\mu / 2} e^{\left(r_{0}^{2}+r^{2}\right) / 4\left(t_{0}-t\right)}
\end{aligned}
$$

Proof. We observe, by definition, that

$$
K_{\mu}\left(r_{0}, \xi ; t_{0}-t^{\prime}\right) \geqq \frac{2 \xi^{\mu-1}}{\Gamma\left(\frac{\mu}{21}\right)\left[4\left(t_{0}-t^{\prime}\right)\right]^{\mu / 2}} e^{-\left(r_{0}^{2}+\xi^{2}\right) / 4\left(t_{0}-t^{\prime}\right)} .
$$


If we apply (4.3) to $K_{\mu}\left(r, \xi ; t-t^{\prime}\right)$, it follows that the left member of (6.6) is bounded by

$$
\left(\frac{t_{0}-t^{\prime}}{t-t^{\prime}}\right)^{\mu / 2} e^{r_{0}^{2} / 4\left(t_{0}-t^{\prime}\right)} \exp \left[\xi^{2} / 4\left(t_{0}-t^{\prime}\right)-(r-\xi)^{2} / 4\left(t-t^{\prime}\right)\right] .
$$

Upon applying the inequality (6.5) to the second exponential (its argument), we obtain the result (6.6).

With these lemmas available, we now have the essential tools for applying the arguments in $\S 6$ of [9] to prove

THEOREM 6.2. Let $u(r, t)$ satisfy the conditions: (i) $u(r, t) \in H, 0<t<c$ and (ii) $\int_{0}^{\infty}|u(r, t)| W_{\mu}(r) S_{\mu}(r, c-t) d r<M$. These are necessary and sufficient that $u(r, t)$ have the Poisson-Stieltjes integral representation

$$
u(r, t)=\int_{0}^{\infty} K_{\mu}(r, \xi ; t) d \alpha(\xi)
$$

where $\int_{0}^{\infty} W_{\mu}(\xi) S_{\mu}(\xi, c)|d \alpha(\xi)|<\infty$.

With this, we conclude our treatment leading up to the proof of our Theorem 6.1. We only note that in proving that any solution $u(r, t)$ of $(1.3)$ which is bounded by $K e^{A r^{2}}$ for $a<t<b$ satisfies the Huygen's principle there, the condition (ii) of Lemma 6.4 translates into

$$
u(0, t)=\int_{0}^{\infty} K_{\mu}\left(0, \xi ; t-t^{\prime}\right) u\left(\xi, t^{\prime}\right) d \xi .
$$

This indicates the structure demanded of $u$ at the singular point $r=0$. In particular, if $r=0$ is a removable singular point, then we can use this condition to redefine $u$ there.

We conclude this section with two theorems. One of these relates the time independence of certain integrals to the Huygen's principle and the other shows that the basic radial heat functions $\left\{R_{j}^{\mu}\right\}$ and $\left\{\tilde{R}_{j}^{\mu}\right\}$ satisfy the Huygen's principle.

THEOREM 6.3. Let $u(r, t) \in H^{*}$ for $a<t<b$ and let $v(r, t) \in H^{*}$ for $a<-t<b$. Assume further that the following condition maintains for $a<t<t^{\prime}<b$ :

$$
\int_{0}^{\infty} W_{\mu}(\xi)|u(\xi, t)| d \xi\left\{\int_{0}^{\infty} K_{\mu}\left(\xi, r ; t^{\prime}-t\right)\left|v\left(r,-t^{\prime}\right)\right| d r\right\}<\infty .
$$

Then the function $g(t)=\int_{0}^{\infty} W_{\mu}(\xi) u(\xi, t) v(\xi,-t) d \xi$ is independent of $t$ in $(a, b)$.

Proof. By writing a similar expression for $g\left(t^{*}\right)$ with, say, $t^{*}>t$ and $a<t^{*}<b$, we can replace $u\left(r, t^{*}\right)$ by $\int_{0}^{\infty} K_{\mu}\left(r, \xi ; t^{*}-t\right) u(\xi, t) d \xi$ (under the hypothesis) in this integral for $g\left(t^{*}\right)$. Upon interchanging the order of integration in this (permissible by the convergence hypothesis), we find that the expression for 
$g\left(t^{*}\right)$ reduces to the one for $g(t)$. The interchange property (3.7) and the condition $v \in H^{*}$ are needed to complete the reduction after this interchange has been made.

THEOREM 6.4. (a) All members of $\left\{R_{j}^{\mu}\right\}$ are in $H^{*}$ for all $t$ (real), and (b) all members of $\left\{\widetilde{R}_{j}^{\mu}\right\}$ are in $H^{*}$ for $t>0$.

Proof. (a). We could give this proof by resorting to integrals of special functions directly. However, let us use the definition (2.3) and the result (3.5b). From (2.3) we have for $t>t^{\prime}$,

$$
\begin{aligned}
& \int_{0}^{\infty} K_{\mu}\left(r, \xi ; t-t^{\prime}\right) R_{j}^{\mu}\left(\xi, t^{\prime}\right) d \xi \\
& =\Gamma\left(\frac{\mu}{2}+j\right) \sum_{k=0}^{j} \frac{1}{\Gamma\left(\frac{\mu}{2}+k\right)}\left(\begin{array}{c}
j \\
k
\end{array}\right)\left(4 t^{\prime}\right)^{j-k} R_{k}^{\mu}\left(r, t-t^{\prime}\right) \\
& =\Gamma\left(\frac{\mu}{2}+j\right) \sum_{k=0}^{j}\left(\begin{array}{c}
j \\
k
\end{array}\right)\left(4 t^{\prime}\right)^{j-k}\left\{\sum_{l=0}^{k} \frac{1}{\Gamma\left(\frac{\mu}{2}+l\right)}\left(\begin{array}{c}
k \\
l
\end{array}\right)\left[4\left(t-t^{\prime}\right)\right]^{k-l} r^{2 l}\right\} \\
& =\Gamma\left(\frac{\mu}{2}+j\right) \sum_{k=0}^{j} \sum_{l=0}^{k} \frac{1}{\Gamma\left(\frac{\mu}{2}+l\right)}\left(\begin{array}{c}
j \\
k
\end{array}\right)\left(\begin{array}{c}
k \\
l
\end{array}\right)\left(4 t^{\prime}\right)^{j-k}\left[4\left(t-t^{\prime}\right)\right]^{k-l} r^{2 l} \\
& =\Gamma\left(\frac{\mu}{2}+j\right) \sum_{l=0}^{k} \frac{r^{2 l}}{\Gamma\left(\frac{\mu}{2}+l\right)}\left\{\begin{array}{c}
j-l \\
\left.\sum_{n=0}\left(\begin{array}{c}
j \\
n+l
\end{array}\right)\left(\begin{array}{c}
n+l \\
l
\end{array}\right)\left(4 t^{\prime}\right)^{j-n-l}\left[4\left(t-t^{\prime}\right)\right]^{n}\right\}
\end{array}\right.
\end{aligned}
$$

The inner sum in this last double sum reduces to

$$
\left(\begin{array}{l}
j \\
l
\end{array}\right)(4 t)^{j-\imath}
$$

so that this last sum gives $R_{j}^{\mu}(r, t)$ by $(2.3)$ and we have proved (a).

(b) This can be proved easily by application of Theorem 6.1. However, let us give a proof by means of a generating function. Let $t>t^{\prime}>0$ and select $a \geqq 0$ with $4 a<t^{\prime}$. Then by (2.1) and (2.5)

$$
\int_{0}^{\infty} K_{\mu}\left(r, \xi ; t-t^{\prime}\right) S_{\mu}\left(\xi, t^{\prime}+4 a\right) d \xi=S_{\mu}(r, t+4 a)=\sum_{j=0}^{\infty} \frac{a^{j}}{j !} \tilde{R}_{j}^{\mu}(r, t),
$$

this last holding by (2.11). However, if we replace $S_{\mu}\left(\xi, t^{\prime}+4 a\right)$ in this integral by its series expansion and interchange the orders of integration and summation, we obtain formally that this integral reduces to 


$$
\sum_{j=0}^{\infty} \frac{a^{j}}{j !} \int_{0}^{\infty} K_{\mu}\left(r, \xi ; t-t^{\prime}\right) \tilde{R}_{j}^{\mu}\left(\xi, t^{\prime}\right) d \xi
$$

and (b) follows by comparing the sums in (6.7) and (6.8). The interchange above is permissible if, say,

$$
\sum_{j=0}^{\infty} \frac{a^{j}}{j !} \int_{0}^{\infty}\left|K_{\mu}\left(r, \xi ; t-t^{\prime}\right)\right| \cdot\left|\tilde{R}_{j}^{\mu}\left(\xi, t^{\prime}\right)\right| d \xi<\infty .
$$

By (4.3) and (4.2b), we can obtain a sum which dominates this. This sum converges if $4 a / t^{\prime}<1$ as is easily shown by the ratio test.

7. The expansion theorems. We are finally in the position to give the basic theorems on the expansions of solutions of (1.3) in terms of the sets $\left\{R_{j}^{\mu}\right\}$ and $\left\{\widetilde{R}_{j}^{\mu}\right\}$. The three theorems given are analogous to Theorems $11.1,12.3$, and 12.1, respectively, of [9]. The third theorem characterizes solutions $u(r, t)$ of $(1.3)$ that have convergent expansions in the set $\left\{\widetilde{R}_{j}^{\mu}\right\}$ in terms of an integral related to the Hankel transform. We have developed, in the preceding sections, the counterparts to all of the essential elements entering into the proofs of Theorems 11.1 and 12.3 of [9]. Therefore, we omit the proofs of the first two theorems given in order to avoid unnecessary duplication of effort.

THEOREM 7.1. A necessary and sufficient condition that

$$
u(r, t)=\sum_{j=0}^{\infty} a_{j} R_{j}^{\mu}(r, t),
$$

the series converging in the strip $|t|<\sigma$, is that $u(r, t) \in H^{*}$ in this strip. Moreover, the coefficients $a_{j}$ in this are given by

(a) $a_{j}=\frac{1}{2^{4 j} j ! \Gamma\left(j+\frac{\mu}{2}\right)} \int_{0}^{\infty} W_{\mu}(\xi) u(\xi,-t) \tilde{R}_{j}^{\mu}(\xi, t) d \xi, \quad 0<t<\sigma$,

(b) $a_{j}=\left.\frac{1}{(2 j) !} \frac{\partial^{2 j}}{\partial r^{2 j}} u(r, 0)\right|_{r=0}$.

THEOREM 7.2. A necessary and sufficient condition that

$$
u(r, t)=\sum_{j=0}^{\infty} b_{j} R_{j}^{\mu}(r, t),
$$

the series converging in the upper half-plane $t>\sigma \geqq 0$, is that $u(r, t) \in H^{*}$ and that $\int_{0}^{\infty} \xi^{\mu-1} e^{\xi^{2} / 8 t}|u(\xi, t)| d \xi<\infty$ in this half-plane. Moreover, we have

$$
b_{j}=\frac{1}{2^{4 j} j ! \Gamma\left(j+\frac{\mu}{2}\right)} \int_{0}^{\infty} W_{\mu}(\xi) R_{j}^{\mu}(\xi,-t) u(\xi, t) d \xi, \quad \sigma<t<\infty
$$


THEOREM 7.3. A necessary and sufficient condition that $u(r, t)$ have the representation (7.3), the series converging for $t>\sigma \geqq 0$, is that

$$
u(r, t)=(2 \pi)^{-\mu / 2} r^{1-(\mu / 2)} \int_{0}^{\infty} \xi^{\mu / 2} J_{\mu / 2-1}(r \xi) e^{-\xi^{2} t} \phi(\xi) d \xi
$$

where $\phi(\xi)$ is an entire function of growth $(1, \sigma)$ in $\xi^{2}$. The expression $(7.5)$ can be written alternatively as

$$
u(r, t)=(2 \pi)^{-\mu / 2} r^{(1-\mu) / 2} \mathscr{H}_{\mu / 2-1}\left(r, r^{(\mu-1) / 2} e^{-r^{2} t} \phi(r)\right)
$$

where $\mathscr{H}_{\mu / 2-1}(r, \psi(r))$ denotes the Hankel transform of $\psi(r)$, namely $\mathscr{H}_{\mu / 2-1}(r, \psi(r))$ $=\int_{0}^{\infty}(r \xi)^{1 / 2} J_{\mu / 2-1}(r \xi) \psi(\xi) d \xi$. Moreover, the $b_{j}$ in (7.3) are given by $b_{j}=(-1)^{j} \phi^{(2 j)}(0) / 4^{j}(2 j) !$.

Proof. Sufficiency. Now we have, by the integral representation (3.5a) and the Appell transform

$$
\tilde{R}_{j}^{\mu}(r, t)=\frac{(4 \pi t)^{-\mu / 2}}{2 t^{2 j+1}} \int_{0}^{\infty} r^{1-\mu / 2} \eta^{\mu / 2} e^{-\eta^{2} / 4 t} J_{\mu / 2-1}\left(\frac{r \eta}{2 t}\right)(i \eta)^{2 j} d \eta
$$

and under the change of variables $\eta=2 \xi t$, this becomes

$$
\tilde{R}_{j}^{\mu}(r, t)=\int_{0}^{\infty}\left(-4 \xi^{2}\right)^{j} r^{1-\mu / 2} J_{\mu / 2-1}(r \xi) e^{-\xi^{2} t}\left(\frac{\xi}{2 \pi}\right)^{\mu / 2} d \xi .
$$

By writing $\phi(\xi)=\sum_{j=0}^{\infty} a_{j} \xi^{2 j}$ and substituting into the integral (7.5) we obtain

$$
\begin{aligned}
u(r, t) & =(2 \pi)^{-\mu / 2} r^{1-\mu / 2} \int_{0}^{\infty} \xi^{\mu / 2} J_{\mu / 2-1}(r \xi) e^{-\xi^{2} t}\left\{\sum_{j=0}^{\infty} a_{j} \xi^{2 j}\right\} d \xi \\
& =\sum_{j=0}^{\infty} r^{1-\mu / 2}(-4)^{-j} a_{j} \int_{0}^{\infty}\left(\frac{\xi}{2 \pi}\right)^{\mu / 2} J_{\mu / 2-1}(r \xi) e^{-\xi^{2} t}\left(-4 \xi^{2}\right)^{j} d \xi \\
& =\sum_{j=0}^{\infty}(-4)^{-j} a_{j} \widetilde{R}_{j}^{\mu}(r, t) \quad(\text { by }(7.5))=\sum_{j=0}^{\infty} b_{j} \widetilde{R}_{j}^{\mu}(r, t)
\end{aligned}
$$

with $b_{j}=(-4)^{-j} a_{j}=\phi^{(2 j)}(0) /(-4)^{j}(2 j)$ !. The above interchange of summation and integration is valid if, say,

$$
\int_{0}^{\infty}\left|\xi^{\mu / 2} J_{\mu / 2-1}(r \xi) e^{-\xi^{2} t}\right| \cdot\left(\sum_{j=0}^{\infty}\left|a_{j}\right| \xi^{2 j}\right) d \xi<\infty
$$

This is dominated by the integral

$$
\int_{0}^{\infty} \xi^{\mu / 2} e^{-\xi^{2} t}\left(\sum_{j=0}^{\infty}\left|a_{j}\right| \xi^{2 j}\right) d \xi
$$

which converges, because the growth condition on $\phi(\xi)$ implies that the inner sum is bounded by $M e^{\xi^{2} t^{\prime}}, t^{\prime} \leqq \sigma<t$, for some $M>0$. 
Necessity. Assume that $\sum_{j=0}^{\infty} b_{j} \tilde{R}_{j}^{\mu}(r, t)$ converges. Take $c>\sigma$. By (2.10) the series $\sum_{j=0}^{\infty}\left\{b_{j} R_{j}^{\mu}(r,-t) / t^{2 j}\right\}$ converges for all $r \geqq 0$ on $t=c$ and by Lemma 5.3, $b_{j}=O\left[(c e / 4 j)^{j+(\mu-1) / 4}\right]$ as $j \rightarrow \infty$.

Then

$$
\begin{aligned}
u(r, t) & =\sum_{j=0}^{\infty} b_{j} \widetilde{R}_{j}^{\mu}(r, t) \\
& =\sum_{j=0}^{\infty} b_{j} \int_{0}^{\infty} r^{1-\mu / 2} J_{\mu / 2-1}(r \xi) e^{-\xi^{2} t}\left(-4 \xi^{2}\right)^{j}\left(\frac{\xi}{2 \pi}\right)^{\mu / 2} d \xi \\
& =\int_{0}^{\infty}\left(\frac{\xi}{2 \pi}\right)^{\mu / 2} r^{1-\mu / 2} J_{\mu / 2-1}(r \xi) e^{-\xi^{2} t} \phi(\xi) d \xi
\end{aligned}
$$

with $\phi(\xi)=\sum_{j=0}^{\infty} b_{j}\left(-4 \xi^{2}\right)^{j}=\sum_{j=0}^{\infty} a_{j} \xi^{2 j}$. We must prove that

$$
\int_{0}^{\infty} \xi^{\mu / 2} e^{-\xi^{2 t}}\left(\sum_{j=0}^{\infty}\left|b_{j}\right|\left(4 \xi^{2}\right)^{j}\right) d \xi<\infty
$$

to give validity to the above representation. But from our growth bound on $b_{j}$ as $j \rightarrow \infty$, we have

$$
\limsup _{j \rightarrow \infty} \frac{j}{e}\left|a_{j}\right|^{1 / j}=\limsup _{j \rightarrow \infty} \frac{j}{e}\left|4^{j} b_{j}\right|^{1 / j} \leqq c<\sigma .
$$

Thus $\phi(\xi)$ is an entire function of growth $(1, \sigma)$ in $\xi^{2}$.

Added in proof. The expansion theorems given here have recently been discovered independently by D.T. Haimo [13]. Her notation and development differ somewhat from that used in this paper.

\section{REFERENCES}

1. P. Appell, Sur l'équation $\partial^{2} z / \partial x^{2}-\partial z / \partial y=0$ et la théorie de la chaleur, J. Math. Pures Appl. 8 (1892), 187-216.

2. L. Bragg, A Rodrigues' formula for the Laguerre polynomials, Amer. Math. Monthly (to appear).

3. H. Buchholz, Die konfluente hypergeometrische Funktion, Springer-Verlag, Berlin, 1953.

4. A. Erdélyi (Ed.), Bateman manuscript project, higher transcendental functions, Vol. II, McGraw-Hill, New York, 1953.

5. E. Feldheim, On Laguerre and Hermite polynomials, Quart. J. Math. Oxford Ser. 11 (1940), 18-29.

6. - Sur les fonctions génératrices des polynomes de Laguerre et d'Hermite, Bull. Sci. Math. (2) 63 (1939), 307-329.

7. I. I. Hirschmann and D. V. Widder, The convolution transform, Princeton Univ. Press, Princeton, N. J., 1955. 
8. E. Rainville, Special functions, Macmillan, New York, 1960.

9. P. Rosenblum and D. V. Widder, Expansions in heat polynomials and associated functions, Trans. Amer. Math. Soc. 92 (1959), 220-266.

10. G. Szegö, Orthogonal polynomials, Amer. Math. Soc. Colloq. Publ. Vol. 23, Amer. Math. Soc., Providence, R. I., 1959.

11. S. Täcklind, Sur les classes quasianalytiques des solutions des équations aux dérivées partielles du type parabolique, Nova Acta Soc. Sci. Upsal. (4) 10 (1936), 1-56.

12. D. V. Widder, Analytic solutions of the heat equation, Duke Math. J. 29 (1962), 497-503.

13. D. T. Haimo, Functions with the Huygens property, Bull. Amer. Math. Soc. 71 (1965), 528-532.

Case Institute of Technology,

Cleveland, OHio 